\title{
INTERACCIONES CON SENTIDO: EFECTOS POSITIVOS DE LA TERTULIA LITERARIA
}

\author{
Roberto Barrientos Mollo
}

La figura 1 muestra una cruda realidad del sistema educativo y es que no existe una equivalencia o coherencia entre lo que sabemos acerca de la mejor manera de aprender y lo que realmente hacemos en las escuelas. Si se preguntase a cualquier docente sobre la mejor manera de aprender, 99\% citaría a Vigotsky y el aprendizaje social, y hasta podría sustentar la importancia de generar abundantes interacciones de aprendizaje entre los estudiantes para lograr mejores aprendizajes. Sin embargo, si acompañamos al salón de clases a esos mismos docentes constataremos que ellos acaparan casi todo el tiempo en el aula explicando y dejando muy pocos espacios para la interacción entre estudiantes con fines de aprendizaje.

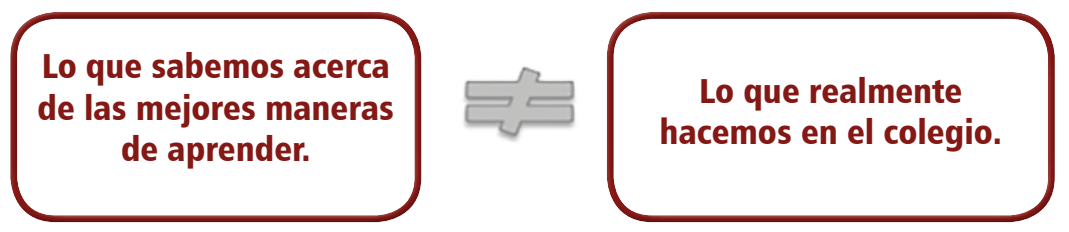

Figura 1. Incoherencias educativas

Un deber importante de los educadores y los decisores de política es cerrar esa brecha entre lo que se sabe y lo que se hace. El presente texto busca ser un aporte en esta tarea. Se propone a las Tertulias Dialógicas Literarias como una estrategia efectiva para garantizar la participación e interacción entre estudiantes para mejores aprendizajes.

\section{La inteligencia colectiva}

El padre de la física social, Alex Pentland, realizó en el año 2010 un estudio con sus colegas en el que descubrieron algo contra todo pronóstico (Woolley, Chabris, Pentland, Hashmi, \& Malone, 2010). Comúnmente se cree que la inteligencia colectiva está dada por el grado de cohesión, motivación y satisfacción de un grupo o hasta por la suma de las inteligencias individuales. Sin embargo, en este estudio se descubrió que uno de los factores que afectaban la inteligencia colectiva era la distribución igualitaria de los turnos de intervención (Ver figura 2). Esta última es una característica esencial de toda Tertulia Dialógica. 

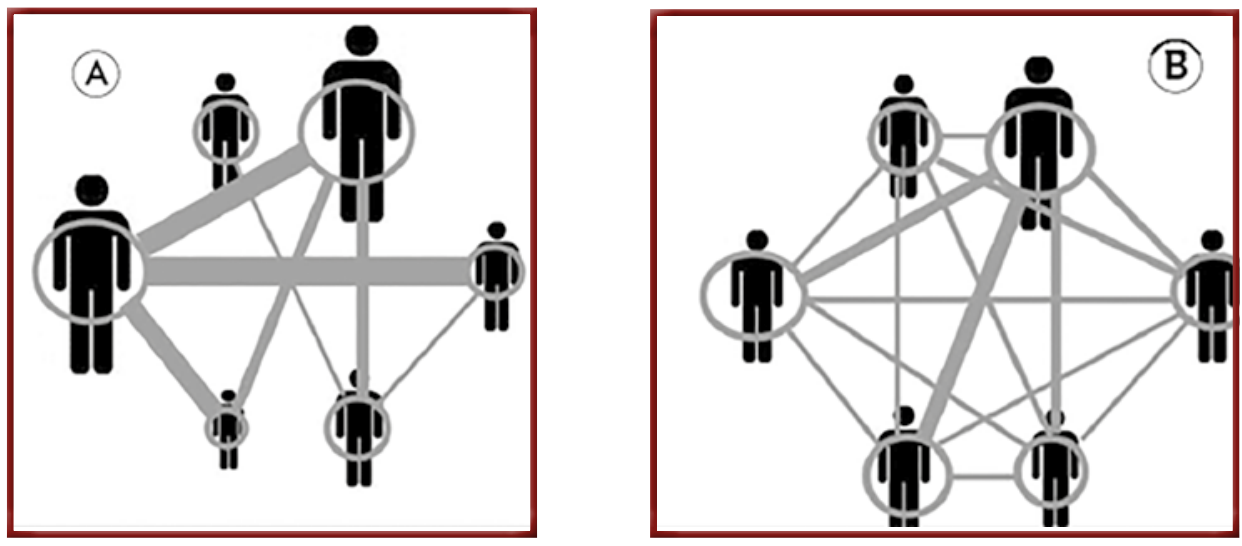

Figura 2. (A) Patrón improductivo de interacción y (B) patrón productivo de interacción. Tomado de Pentland, 2015, p. 89.

Pentland y su equipo del MIT realizaron dos estudios con 699 personas. En cada grupo trabajaban de dos a cinco personas con distintas estrategias de trabajo en equipo. Los investigadores descubrieron que, efectivamente, existe un factor de inteligencia colectiva general, llamado factor " $c$ ", que explica el desempeño de un grupo en una amplia variedad de tareas. Este "factor $c$ " se correlaciona con tres elementos: a) la sensibilidad social promedio de los miembros del grupo, b) la distribución igualitaria del turno de conversación y c) la proporción de mujeres en el grupo. Posteriormente Pentland y su equipo ha realizado otros estudios a gran escala sobre el poder de las interacciones utilizando información de diversas fuentes digitales, al punto de ser uno de los principales exponentes de lo que se conoce como la Física Social. Lo que muestran los descubrimientos de Pentland es que las interacciones son más potentes de lo que se había pensado y tienen un alto poder para modelar nuestra vida personal y nuestra vida en sociedad (Pentland, 2015).

\section{¿Qué se ha investigado acerca de las interacciones en educación?}

A continuación se presenta la revisión de cuatro estudios sobre interacciones en el aula. Realizados en los años 60, 80, 90 y 2016; éste último sobre las Tertulias Literarias Dialógicas (Flanders, 1961, Galton, 1980, 1999, Hargreaves y Gacía-Carrión, 2016).

Net Flanders y su equipo en la universidad de Minnesota en Estados Unidos descubrió que dos tercios de la conversación en el aula estaba acaparada por el docente (Flanders, 1961). Con una muestra de 147 profesores de todos los niveles escolares llegaron a registrar un millón 250 mil interacciones. El estudio duró seis años. En dicho trabajo se clasificaron declaraciones verbales dentro de 10 categorías una vez cada tres segundos. Las categorías eran: 
1. Clarifica constructivamente

2. Exhorta

3. Clarifica, usando ideas sugeridas por los estudiantes

4. Realiza preguntas

5. Exposición docente

6. Ofrece orientaciones

7. Ofrece críticas

8. Intervención del estudiante en respuesta al docente

9. Intervención del estudiante iniciado por el estudiante

10. Silencio o confusión (Flanders, 1961, p. 56).

El sistema que se desarrolló fue denominado "Análisis de interacción", que si bien fue concebido para la investigación, devino en una herramienta útil para la formación continua docente. El principal resultado fue que se descubrió un patrón de interacciones que llamaron la "regla de los dos tercios". Esta regla se subdivide en tres partes: 1) Cerca del 2/3 del tiempo invertido en la clase habla o domina la conversación alguien, 2) Existen 2 de tres posibilidades que ese alguien sea el profesor y 3) En esos dos tercios del tiempo que ocupa el docente: a) expresa sus propias opiniones, b) ofrece orientaciones y c) ofrece críticas a los estudiantes. En años superiores se mantiene el patrón, salvo que, en la tercera parte de la regla, el 40\% de todas las intervenciones docentes son: a) exposición, b) ofrecer directrices y c) ofrecer críticas. Lo que mostró este estudio es que los docentes ocupaban buena parte del tiempo del salón de clases explicando.

Años después se realizó otro estudio icónico sobre el tema en el Reino Unido (Galton \& Simon, 1980). Maurice Galton, docente de la universidad de Leicester, y su equipo realizaron el primer estudio experimental a gran escala en aulas de primaria, llamado proyecto ORACLE (Observational Research And Classroom Learning Evaluation). Dicho proyecto inició en 1975 y tuvo una duración de tres años. Contó con 58 escuelas participantes de tres distintas localidades escolares (Local Authories). Se registraron las interacciones cada 25 segundos y se utilizaron tres instrumentos: a) Registro del alumno, en el que se consignan las actividades de la clase de un alumno a la vez, especialmente de su interacción con el profesor, otros alumnos, las actividades que persiguió como parte de su trabajo, y su ubicación, b) Registro del maestro en cuanto a los tipos de preguntas que se utilizan, y las declaraciones realizadas, sumadas al registro de interacción silenciosa, tales como gestos detalles y, por último, c) una hoja de resumen que ofrecía descripciones 
de la forma de aula y actividades realizadas, así como procedimientos utilizados por los profesores.

Los resultados de este estudio fueron similares al estudio de Flanders, el alumno promedio está 2/3 horas de clase trabajando solo por su propia cuenta. La atención efectiva individual por parte del docente se produce solo a 2,3\% del tiempo de los estudiantes en el aula. En la muestra había escuelas progresistas con enfoques avanzados de pedagogía, sin embargo mostraban los mismos resultados de acaparación de tiempo por parte del docente. Lo que llamó la atención de los investigadores en ese momento es la poca interacción entre estudiantes: "Lo que no se esperaba en ese momento era que también trabajaran con una cantidad muy limitada de contacto con otros alumnos cuando el maestro estaba ocupado en otro lugar" (Galton et. al, 1999, p.79).

Veinte años después Galton y su equipo tuvieron la oportunidad de realizar la misma investigación. La pregunta de investigación fue: "Dadas las mismas escuelas, los mismos instrumentos de investigación y los mismos métodos de análisis, ¿ha cambiado la práctica?" (1999, p. 33). Los resultados mostraron que la proporción de interacción docente- alumnos se ha mantenido igual (Galton, 1999). Afirman los autores:

Lo que es notable, sin embargo, es que cuando hicimos la misma comparación para el registro del estudiante, el patrón o distribución de esas interacciones ha permanecido virtualmente idéntico. Por lo tanto, aunque los niveles de interacción en general pueden ser más altos ahora que antes, encontramos que para el alumno típico, el 75 por ciento de todos los intercambios de maestros-alumnos son experimentados como miembros de la clase, exactamente como lo eran hace veinte años (Galton et. al. p.84).

Como se puede ver en las tres investigaciones revisadas, la hegemonía docente en los salones de clase, expresada en el tiempo que se ocupa en hablar, es un problema que no ha mejorado con el paso de los años. Se ha realizado una revisión de estudios icónicos en el tema de los años 60, 80 y 90 para que evidenciar que no existen muchos avances al respecto.

A manera de corolario quisiera citar afirmaciones recientes del investigador de la Facultad de Educación de la universidad de Harvard, Richard Elmore sobre la acaparación del tiempo por parte de los docentes:

En los salones que observé en escuelas norteamericanas encontré un patrón bastante estable de 85-90 \% de habla de adultos y 10-15 porciento de habla de estudiantes. Los adultos en general 
hablaban en largos y ampulosos períodos, frecuentemente con frases o párrafos incompletos. Los estudiantes, por lo general, hablaban brevemente, emisiones de menos de doce palabras; también en frases incompletas, frecuentemente para terminar con dos o tres palabras la frase que habían iniciado los adultos. Si la teoría del desarrollo del cerebro considera que hablar produce aprendizaje, lo que yo observé sugiere que las oportunidades de desarrollo mental habían sido mínimas (Elmore, 2016, p.15).

Entonces, lo que viene ocurriendo es que se ofrece en las escuelas pocas oportunidades para el desarrollo mental de los estudiantes, dadas las pocas oportunidades de interacción, como recomienda la teoría de aprendizaje social (Vygotski, 1999).

¿Qué dice la literatura al respecto en escuelas peruanas? Se hizo la revisión y, hasta el año 2017, no se han encontrado estudios sobre el tema.

Las Tertulias Literarias Dialógicas son una estrategia para dar solución a esta brecha de oportunidades. A continuación se realiza una breve explicación de su funcionamiento y luego se muestran los resultados de un reciente estudio realizado utilizando las herramientas del proyecto ORACLE en el Reino Unido (Hargreaves \& García-Carrión, 2016).

\section{Las Tertulias Literarias Dialógicas}

Las Tertulias Literarias son encuentros de discusión semanales que ocurren durante el horario de clases en torno a una obra clásica de la literatura universal en los que se fomenta el diálogo igualitario. El docente con el conjunto de estudiantes eligen la obra a leer y acuerdan un número de páginas de lectura entre cada tertulia. Los estudiantes deben de seleccionar un párrafo o frase que les llamó más atención. Al inicio de la tertulia la clase se distribuye en forma de círculo (ver figura 3). Luego el moderador invita a los participantes a realizar intervenciones, para lo cual anota los turnos de palabra. Una vez iniciados los turnos de palabras, otros estudiantes pueden realizar intervenciones espontáneas. Se invita a que cada intervención debe tener la debida argumentación. Al finalizar la Tertulia el moderador con el grupo acuerdan el número de páginas a leer para la próxima tertulia. En el año 2017 esta práctica se encuentra difundida en 100 Instituciones Educativas en 16 regiones del Perú, como parte del proyecto Comunidades de Aprendizaje. 

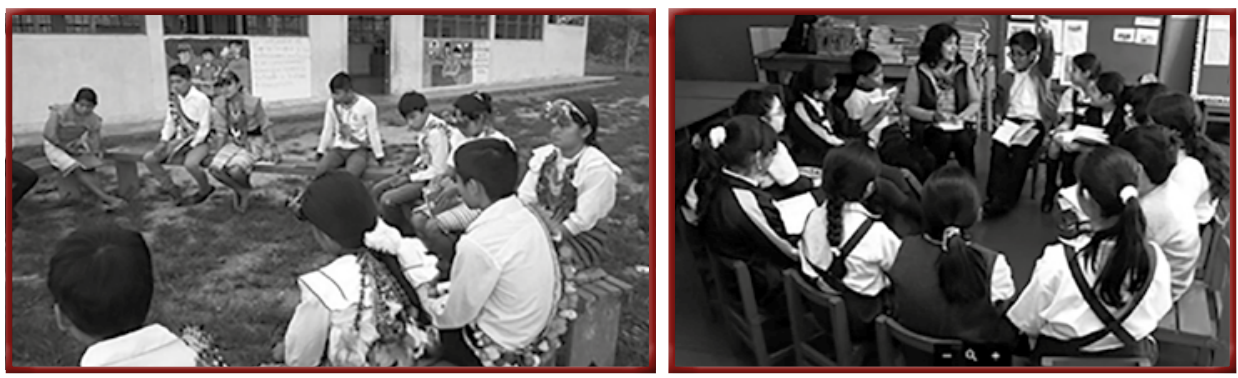

Figura 3. Realización de Tertulia Literaria en el CRFA Balsapuerto de la comunidad Shayahuita de Puerto Porvenir, provincia de Alto Amazonas (izquierda) y en el Colegio María Parado de Bellido, Barrios Altos - Cercado de Lima (derecha)

El año 2015, las investigadoras de la universidad de Cambridge, Linda Hargreaves y Rocío García-Carrión decidieron realizar una medición del impacto de las Tertulias Literarias Dialógicas utilizando los instrumentos del proyecto ORACLE en cuatro escuelas primarias (2016) . El estudio fue guiado por las siguientes preguntas de investigación: ¿La proporción de conversación entre alumno y maestro se ha desplazado hacia más conversaciones con los alumnos? ¿Tienen los alumnos la oportunidad de expresarse ampliamente? ¿Qué proporción de toda la clase contribuye al diálogo? ¿Los alumnos fundamentan y discuten las ideas de los demás?

El resultado más llamativo de esta investigación fue que la proporción de conversación de alumnos con maestros se mejora drásticamente en comparación con la regla de dos tercios del estudio de Flanders y el estudio del proyecto ORACLE. En las Tertulias Literarias Dialógicas analizadas las proporciones oscilan entre el $75 \%$ y el $97 \%$ del tiempo de conversación entre los alumnos, con una media del 85\% (Hargreaves y García-Carrión, 2016). Por lo que se puede afirmar que las Tertulias Literarias Dialógicas son una estrategia efectiva para generar espacios constructivos de diálogo entre los estudiantes.

\section{Conclusiones}

La interacción estudiante-estudiante y docente-estudiante son dos tópicos que necesitan ser profundizados para poder favorecer el desarrollo mental de los estudiantes. La Tertulia Literaria contribuye a generar una mayor cantidad y calidad de interacciones con sentido, favoreciendo el diálogo igualitario, por lo que se recomienda fomentar su difusión en escuelas y otros ambientes comunitarios. Así mismo, el presente escrito es un llamado a generar más estrategias que ayuden disminuir el rol hegemónico del docente en el aula y que empoderen a los estudiantes de su propio proceso de aprendizaje, mediante la construcción participativa de significados. 


\section{REFERENCIAS}

Elmore, R. (2016). Reflexiones sobre la contribución de la Tutoría al futuro del aprendizaje. Consultado en: http://redesdetutoria.com/wp-content/uploads/2017/03/futurodel aprendizaje2.pdf

Flanders, N. A. (1961). Analyzing teacher behavior. Addison-Wesley P. C.

Galton, M. J. (1999). Inside the primary classroom: 20 years on: Psychology Press.

Galton, M. J., \& Simon, B. (1980). Progress and performance in the primary classroom: Routledge.

Hargreaves, L., \& García-Carrión, R. (2016). Toppling Teacher Domination of Primary Classroom Talk through Dialogic Literary Gatherings in England. Paper presented at the FORUM: for promoting 3-19 comprehensive education.

Pentland, A. (2015). Social Physics: How Social Networks Can Make Us Smarter. Penguin.

Vygotski, L. S. (1999). Pensamiento y lenguaje: teoría del desarrollo cultural de las funciones psíquicas: Fausto.

Woolley, A. W., Chabris, C. F., Pentland, A., Hashmi, N., \& Malone, T. W. (2010). Evidence for a collective intelligence factor in the performance of human groups. science, 330(6004), 686-688. 